\title{
Perfil clínico dos pacientes atendidos em um ambulatório de fisioterapia em oncologia do município de São Paulo
}

Clinical profile of patients assisted in a physical therapy outpatient service in oncology of Sao Paulo city

\author{
Pascale Mutti Tacani* \\ Karina Tamy Kasawara** \\ Rogério Eduardo Tacani*** \\ Aline Fernanda Peres Machado**** \\ Débora Montezello***** \\ João Carlos Guedes Sampaio Góes******
}

\begin{abstract}
Resumo
Os avanços no tratamento das neoplasias têm favorecido maiores taxas de sobrevida, aumentando a demanda da reabilitação dos pacientes com câncer, sendo a fisioterapia uma das áreas em amplo crescimento. Porém, essa população possui perfil, demanda e realidade bem diferentes, sendo necessário o conhecimento de suas características para melhor elaborar estratégias de atuação e condutas dentro da área de fisioterapia em oncologia. O objetivo deste estudo foi identificar o perfil clínico dos pacientes atendidos em um ambulatório de fisioterapia em oncologia. Foram analisados 105 prontuários para a coleta dos dados sociodemográficos e clínicos de um ambulatório de fisioterapia em oncologia do município de São Paulo, de agosto de 2008 a julho de 2010, sendo 11 $(10,5 \%)$ excluídos. Para a análise estatística foi utilizado o teste de igualdade de duas proporções para avaliar a distribuição relativa das variáveis e Mann-Whitney para comparação destas. Dos 94 prontuários analisados, $63,8 \%(n=60)$ eram do gênero feminino ( $p<0,001$ ), com faixa etária média de 56,3 $\pm 13,3$ anos e IMC de 27,09 $\pm 5,88 \mathrm{~kg} / \mathrm{m}^{2}$. O diagnóstico médico mais frequente foi o câncer de mama em 44,4\% ( $n=44 ; p<0,05)$. A maioria estava entre o segundo e quinto ano (33\%; $n=31)$ de evolução da doença. O tratamento cirúrgico foi o mais realizado $(94,7 \% ; n=89 ; p<0,001)$. O diagnóstico fisioterapêutico mais encontrado foi de alterações vasculares e linfáticas $(87,2 \% ; n=82 ; p<0,001)$. A queixa principal dos pacientes foi de dor $(55,3 \% ; n=52)$ e edema $(51,1 \% ; n=48 ; p=0,559)$. Pode-se concluir que o perfil clínico dos pacientes se caracterizou por mulheres acima de 50 anos, com sobrepeso, em pós-operatório tardio de câncer de mama, apresentando dor e linfedema nos membros.
\end{abstract}

Palavras-chave: Neoplasias. Perfil de Saúde. Fisioterapia.

\begin{abstract}
Advances in cancer treatment has been favoring higher survival rates, increasing demand of rehabilitation of cancer patients, being physical therapy one of the areas in a higher growth. However, this population has very different profiles, demands and realities, requiring the knowledge of its features to better develop action strategies and conducts in the area of physical therapy in oncology. The objective of this study was to identify the clinical profile of patients assisted in a physical therapy outpatient in Oncology. One hundred five medical records were analyzed for demographic and clinical data collection in a outpatient physiotherapy service of Sao Paulo city, from August 2008 to July 2010, being 11(10.5\%) excluded. Statistical analysis was performed using the test for equality of two proportions to evaluate the relative distribution of variables, which were compared using the Mann-Whitney test. Among those 94 medical records, $63.8 \%(n=60)$ were female $(p<0.001)$, with a mean age of $56.3 \pm 13.3$ years and they had a BMI of $27.09 \pm 5.88$ $\mathrm{kg} / \mathrm{m}^{2}$. The most prevalent medical diagnosis was breast cancer in $44.4 \%(\mathrm{n}=44 ; \mathrm{p}<0.05)$. Most patients were between the second and the fifth year $(33 \% ; n=31)$ of disease evolution. Surgery was the most common treatment $(94.7 \% ; n=89 ; p<0.001)$. The most frequent physiotherapeutic diagnosis included vascular and lymphatic dysfunctions $(87.2 \% ; n=82 ; \mathrm{p}<0.001)$ with complaints of pain $(55.3 \% ; n=52)$ and swelling $(51.1 \% ; n=48 ; p=0.559)$. It was concluded that the clinical profile of patients was characterized by woman, over 50 years old, overweight in late postoperative breast cancer, exhibiting lymphedema and pain in the limbs.
\end{abstract}

Keywords: Neoplasms. Health Profile. Physical Therapy Specialty.

\footnotetext{
DOI: $10.15343 / 0104-7809.20143804439447$

* Instituto Brasileiro de Controle do Câncer (IBCC), São Paulo-SP, Brasil. E-mail: pascale.tacani@hotmail.com

** Departamento de Tocoginecologia da Faculdade de Ciências Médias da Universidade Estadual de Campinas (UNICAMP), Campinas-SP, Brasil. E-mail: karina.tamy@gmail.com

*** Centro Universitário São Camilo, São Paulo-SP, Brasil. E-mail: rtacani@uol.com.br

**** Universidade Federal de São Paulo, São Paulo-SP, Brasil. E-mail: lifpm@yahoo.com.br

***** Instituto Brasileiro de Controle do Câncer (IBCC), São Paulo-SP, Brasil. E-mail: debora@ibcc.org.br

****** Instituto Brasileiro de Controle do Câncer (IBCC), São Paulo-SP São Paulo-SP, Brasil. E-mail: clinica-adm@sampaiogoes.com

Os autores declaram não haver conflitos de interesse.
} 


\section{INTRODUÇÃO}

O câncer vem sendo considerado um evidente problema de saúde pública mundial. A Organização Mundial da Saúde (OMS) estima que, em 2030, serão 27 milhões de casos incidentes de câncer, 17 milhões de mortes por câncer e que serão diagnosticadas 75 milhões de pessoas, anualmente, com câncer, sendo que o maior efeito desse aumento vai incidir em países de baixa e média renda ${ }^{1}$.

No Brasil, os dados do Instituto Nacional de Câncer (INCA) estimam a ocorrência de 576 mil casos novos de câncer, incluindo os casos de pele não melanoma para o biênio 2014/2015². O câncer de pele não melanoma (182 mil casos novos) é o mais frequente no país, seguido pelo de próstata (69 mil) e de mama (57 mil). A assistência aos pacientes com câncer representa significativo impacto financeiro ao sistema público de saúde e aos sistemas privados ${ }^{3}$.

Por esse motivo, projetos de prevenção e tratamento vêm sendo implantados para meIhorar a saúde pública. Estudos relacionam a prevenção e controle do câncer à mudança de estilo de vida, como, por exemplo, redução do tabagismo, alimentação com redução de produtos de origem animal e aumento no consumo de frutas e vegetais, além da recomendação para a realização de atividade física ${ }^{4,5}$.

Nesse contexto, a fisioterapia é indicada para assistência e promoção da saúde, essencial para manutenção e melhora da qualidade de vida. A prática clínica da fisioterapia vem sendo baseada em evidência com o objetivo de promover saúde ao longo da vida de todos os indivíduos, não somente para prevenção, mas também para controle e tratamento de doenças ${ }^{6}$.

A fisioterapia é descrita em diversos estudos como parte fundamental do tratamento pós-operatório dos diversos tipos de cânceres, promovendo melhora na saúde física e na qualidade de vida desses pacientes ${ }^{7,8}$.

No Brasil, em maio de 2008, foi fundada a Associação Brasileira de Fisioterapia em Oncologia (ABFO), com o intuito de incentivar o desenvolvimento técnico e científico dos fisioterapeutas comprometidos com a assistência, pesquisa, ensino e políticas de saúde em oncologia. Com o desenvolvimento da área, o Conselho Federal de Fisioterapia e Terapia Ocupacional reconheceu a Fisioterapia Oncofuncional como especialidade do profissional fisioterapeuta, conforme Resolução n 364, de 20 de maio de $2009^{9}$.

Para o crescimento e desenvolvimento da profissão e da área de atuação da fisioterapia em oncologia, é preciso conhecer as características dos pacientes a serem atendidos, fundamental para delinear estratégias, atenção e atendimento especializado ${ }^{7}$. Por esse motivo, o objetivo deste estudo foi identificar o perfil clínico dos pacientes atendidos em um ambulatório de fisioterapia em oncologia.

\section{MÉTODO}

Trata-se de um estudo retrospectivo feito a partir da análise de 105 prontuários de pacientes atendidos em um ambulatório de fisioterapia em oncologia do município de São Paulo, no qual o atendimento era feito por estagiários a pacientes do Sistema Único de Saúde (SUS). O período de coleta foi de agosto de 2008 a julho de 2010 e teve início após aprovação do Comitê de Ética do Centro Universitário São Camilo (n 31/10), sendo mantido o sigilo e o anonimato das informações. Os dados foram coletados a partir dos prontuários eletrônicos e também pela ficha de avaliação fisioterapêutica utilizada exclusivamente no estágio de fisioterapia. Nessa verificação foram colhidas: identificação do paciente, data de nascimento, idade, gênero, índice de massa corpórea (IMC), escolaridade, diagnóstico médico, tempo de evolução da doença, tratamento médico, diagnóstico fisioterapêutico e queixas.

O tempo de evolução da doença referiu-se ao período transcorrido entre o conhecimento do diagnóstico até o início da fisioterapia. O tratamento médico foi categorizado em cirúrgia, quimioterapia, radioterapia e demais terapias medicamentosas (Anti-inflamatórios, analgésicos, anti-helméticos, diuréticos, entre outros, incluindo a hormonioterapia). 
O diagnóstico fisioterapêutico foi categorizado em alterações vasculares e linfáticas, cutâneas, musculoesqueléticas e neurológicas, conforme classificação dos quadros após a coleta de dados pela ficha de avaliação fisioterapêutica. A ficha continha a anamnese e o exame físico, composto por dados antropométricos, inspeção, palpação, escala visual analógica de dor, perimetria, testes de sensibilidade superficial, goniometria e teste manual de força muscular. Para esse estudo, dentre o diagnóstico de alterações vasculares e linfáticas, considerou-se edema quando havia inchaço de consistência aquosa à palpação, e linfedema quando havia inchaço de consistência fibrosa, porém não foram classificados quanto à sua gravidade.

O critério de inclusão foi o de selecionar todos os prontuários dos pacientes atendidos no estágio de fisioterapia de agosto de 2008 a julho de 2010 e o de exclusão foi retirar da amostra os prontuários cujas informações estivessem incompletas em três ou mais itens e aqueles, nos quais, registravam que o paciente não havia realizado o tratamento fisioterapêutico.

Os dados foram descritos em porcentagem, média e desvio padrão para faixa etária e IMC. A análise estatística foi realizada utilizando o Teste de Igualdade de Duas Proporções para avaliar a distribuição relativa das variáveis: número de prontuários, gênero, escolaridade, diagnóstico médico, tempo de evolução da doença, tratamento médico, diagnóstico fisioterapêutico e queixa, e Mann-Whitney para comparação entre gênero e faixa etária e entre gênero e IMC. O nível de significância foi estipulado em 5\% ( $p$ $\leq 0,05)$.

\section{RESULTADOS}

Foram analisados 105 prontuários e 94 $(89,5 \%)$, foram incluídos. A descrição de gênero, faixa etária, IMC e nível de escolaridade encontram-se na Tabela 1.

Tabela 1. Distribuição das variáveis: faixa etária, gênero, Índice de Massa Corporal (IMC) e nível de escolaridade. São Paulo, Agosto de 2008 a Julho de 2010

\begin{tabular}{|c|c|c|c|c|}
\hline \multirow{2}{*}{ Gênero } & Geral & Feminino & Masculino & \\
\hline & $n=94$ & $63,8 \%(n=60)$ & $36,2 \%(n=34)$ & $<0,001^{*}$ \\
\hline $\begin{array}{l}\text { Faixa Etária } \\
\text { (Anos) }\end{array}$ & $56,3 \pm 13,3$ & $57,4 \pm 13,1$ & $54,4 \pm 13,8$ & $0,149^{* *}$ \\
\hline \multirow[t]{5}{*}{$\begin{array}{l}\mathrm{IMC} \\
\left(\mathrm{Kg} / \mathrm{m}^{2}\right)\end{array}$} & $27,09 \pm 5,88$ & $29,3 \pm 5,6$ & $23,5 \pm 4,3$ & $<0,001^{* *}$ \\
\hline & & $\mathrm{n}$ & $\%$ & $\mathrm{p}$ - valor \\
\hline & Não Informado & 34 & 36,2 & Ref.* \\
\hline & Ensino fundamental completo & 21 & 22,3 & 0,037 \\
\hline & Ensino fundamental incompleto & 17 & 18,1 & 0,005 \\
\hline \multirow[t]{5}{*}{ Escolaridade } & Ensino médio completo & 14 & 14,9 & $<0,001$ \\
\hline & Ensino médio incompleto & 5 & 5,3 & $<0,001$ \\
\hline & Ensino superior completo & 2 & 2,1 & $<0,001$ \\
\hline & Ensino superior incompleto & 1 & 1,1 & $<0,001$ \\
\hline & Analfabeto & 0 & 0 & $<0,001$ \\
\hline
\end{tabular}

Valores de $\mathrm{p}<0,05$ considerados significantes. *Teste de Igualdade de Duas Proporções; **Teste de Mann-Whitney. 
Os diagnósticos, tempo de evolução da doença e tratamento médico estão apresentados na Tabela 2.

Tabela 2. Distribuição do diagnóstico médico, tempo de evolução da doença e tratamento médico dos 94 prontuários analisados. São Paulo, Agosto de 2008 a Julho de 2010

\begin{tabular}{|c|c|c|c|c|c|}
\hline & & $\mathbf{n}$ & $\%$ & \multicolumn{2}{|c|}{ p - valor } \\
\hline \multirow{8}{*}{ Neoplasia } & Mama & 44 & 44,4 & $<0,05^{1}$ & \\
\hline & Cabeça e Pescoço & 27 & 27,3 & & \\
\hline & Útero & 13 & 13,1 & & \\
\hline & Melanoma & 6 & 6,1 & & \\
\hline & Tecidos Moles & 4 & 4 & & \\
\hline & Vulva & 2 & 2 & & \\
\hline & Ovário & 1 & 1 & & \\
\hline & Próstata & 1 & 1 & & \\
\hline \multirow{9}{*}{$\begin{array}{l}\text { Tempo de evolu- } \\
\text { ção da doença }\end{array}$} & 1 a 3 meses & 0 & 0,0 & & \\
\hline & 4 a 6 meses & 1 & 1,1 & & \\
\hline & 7 a 9 meses & 1 & 1,1 & & \\
\hline & 10 a 12 meses & 7 & 7,4 & & \\
\hline & 13 meses a 2 anos & 20 & 21,3 & & \multirow{2}{*}{$0,071^{* *}$} \\
\hline & 3 a 5 anos & 31 & 33 & $<0,05^{*}$ & \\
\hline & 6 a 10 anos & 19 & 20,2 & & \\
\hline & 11 a 20 anos & 12 & 12,8 & & \\
\hline & 21 a 30 anos & 0 & 0,0 & & \\
\hline \multirow{4}{*}{$\begin{array}{l}\text { Tratamento } \\
\text { médico }\end{array}$} & Cirúrgico & 89 & 94,7 & $<0,001^{*}$ & \\
\hline & Medicamentoso & 16 & 17 & & \\
\hline & Quimioterapia & 52 & 55,3 & & \\
\hline & Radioterapia & 59 & 62,8 & & \\
\hline
\end{tabular}

Valores de $\mathrm{p}<0,05$ considerados significantes. Teste de Igualdade de Duas Proporções. * Diferença significante entre as demais variáveis; ** Diferença entre o período de 2 a 5 anos e 1 a 2 anos.

Os diagnósticos fisioterapêuticos e seus respectivos subtipos foram categorizados em alterações vasculares e linfáticas, cutâneas, musculoesqueléticas, neurológicas e não informado, conforme Tabela 3. 
Tabela 3. Distribuição do diagnóstico fisioterapêutico e respectiva classificação dos 94 prontuários analisados. São Paulo, Agosto de 2008 a Julho de 2010

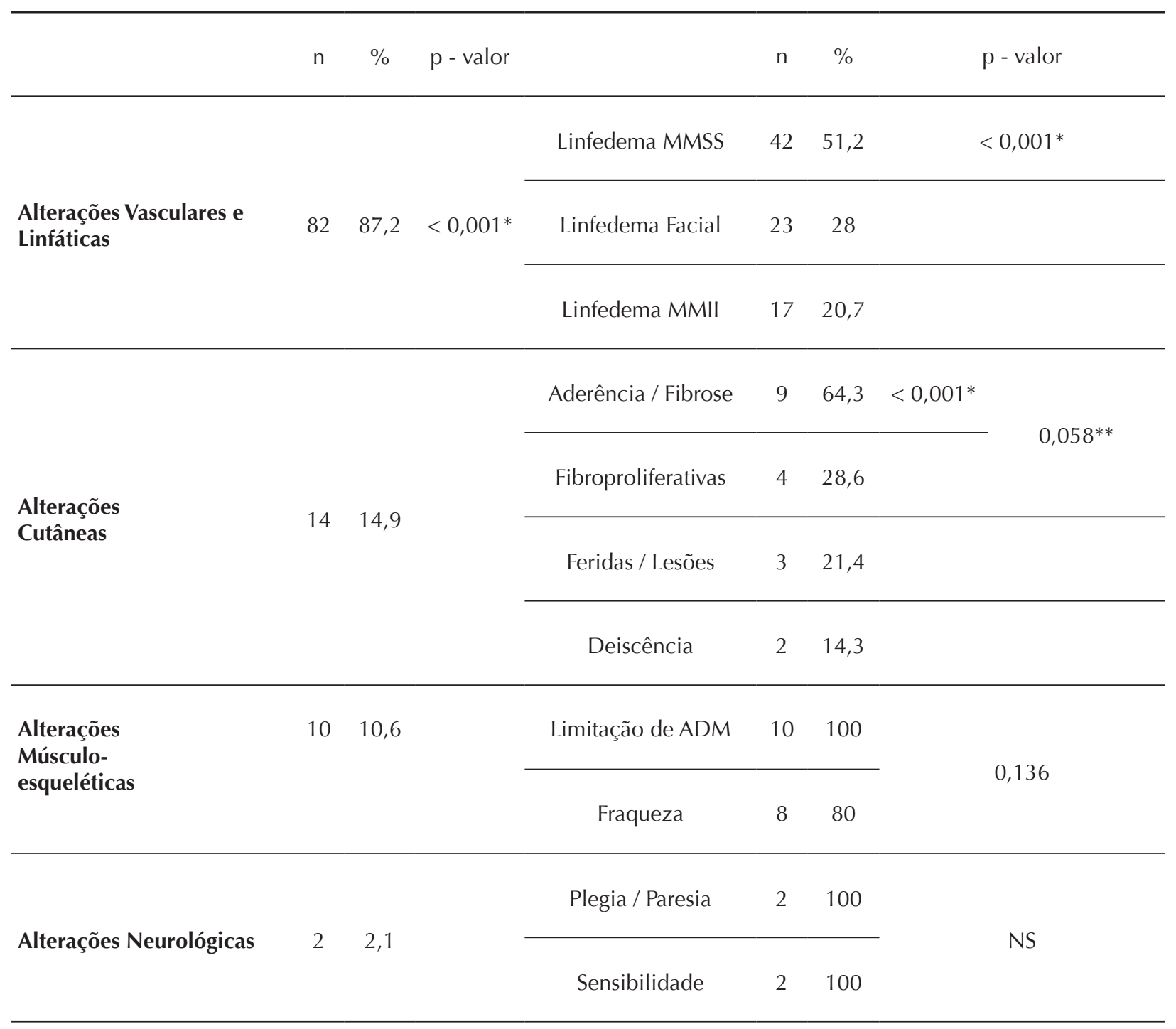

Não Informado

$4 \quad 4,1$

Valores de $\mathrm{p}<$ 0,05 considerados significantes; Teste de Igualdade de Duas Proporções. * Valor de p na comparação com as demais variáveis; ${ }^{* *}$ Valor de $p$ entre Aderência e Fibroproliferativas. NS: Diferença não significante.

Legenda: ADM: Amplitude de Movimento; MMII: Membros Inferiores. MMSS: Membros Superiores.

Nota: Linfedema Facial envolve a região facial e cervical; Fibroproliferativas: se refere às cicatrizes hipertróficas e queloides; Feridas / Lesões se refere a diversos tipos de feridas e Úlcera de Marjolin; Sensibilidade se refere à hipoestesia e anestesia devido à alteração neurológica.

As queixas principais foram categorizadas em queixas relacionadas à dor, edema, disfunção articular, disfunção muscular e disfunção cicatricial ou cutânea, de acordo com a Tabela 4. 
Tabela 4. Queixas dos pacientes categorizadas em 5 aspectos. São Paulo, Agosto de 2008 a Julho de 2010

\begin{tabular}{lllll}
\hline Queixa relacionada & $\mathbf{n}$ & $\%$ & \multicolumn{2}{c}{$\mathbf{p}$ - valor } \\
\hline Dor & 52 & 55,3 & $<0,001^{*}$ \\
\hline Edema & 48 & 51,1 & $<0,001^{*}$ & $0,559^{* *}$ \\
\hline Disfunção Articular & 14 & 14,9 & \\
\hline Disfunção Muscular & 13 & 13,8 & \\
\hline Disfunção Cicatricial ou Cutânea & 0 & 0 & \\
\hline
\end{tabular}

Valores de $\mathrm{p}<0,05$ considerados significantes. Teste de Igualdade de Duas Proporções.

* Valor de p na comparação com as demais variáveis.

** Valor de p na comparação entre Dor e Edema.

*** Valor de p na comparação entre Disfunção Articular e Muscular.

\section{DISCUSSÃO}

O presente estudo verificou o perfil clínico de pacientes atendidos em um ambulatório de fisioterapia, cuja demanda é a de indivíduos encaminhados com diagnóstico de diversos tipos de câncer, porém, como observado por este estudo, a maioria dos pacientes atendidos foram mulheres, com o diagnóstico de câncer de mama, procurando a fisioterapia no período pós-operatório. Apesar da abrangência de diagnósticos e encaminhamentos, o serviço oferecido não abordava todas as subáreas da fisioterapia, dificultando traçar o perfil dos pacientes com necessidades respiratórias, uroginecológicas e hematológicas.

Para analisar as características clínicas e sociodemográficas dos pacientes atendidos no ambulatório de fisioterapia, foi delimitado metodologicamente o estudo do tipo retrospectivo, considerado desenho de estudo ideal para compreender e analisar os fatores de exposição pré$\operatorname{vios}^{7,10}$, ou seja, ideal para conhecer o perfil dos pacientes que foram atendidos no ambulatório. Estudos retrospectivos são essenciais para delimitar diretrizes para que outros tipos de estudos sejam desenvolvidos, como os estudos do tipo ensaio controlado e os estudos prospectivos ${ }^{10}$.

No entanto, a metodologia de coleta retrospectiva dos dados por revisão dos prontuários pode trazer alguns vieses, uma vez que alguns prontuários foram excluídos por falta de informações ${ }^{7,10}$. Da mesma maneira, Tacani, et $\mathrm{al}^{7}$ coletaram informações de 32 prontuários de pacientes com diagnóstico de câncer de cabeça e pescoço, em São Paulo-SP, Brasil, sendo que $23 \%(n=6)$ teve de ser excluído por dados incompletos. Mesmo com dados excluídos, o presente estudo teve uma grande abrangência de dados com 89,5\% de prontuários analisados.

Com relação às características da amostra, neste estudo, as mulheres prevaleceram, representando $63,8 \%$ da amostra. Outros estudos também indicaram que mulheres procuram mais os serviços de saúde do que os homens ${ }^{11}$. As mulheres apresentam uma tendência a ter maior cuidado com o seu estado de saúde, consequentemente à adesão ao tratamento adequado e complementar ao tratamento médico ${ }^{12}$. Diversos fatores podem estar associados à dificuldade de adesão dos pacientes ao tratamento, como fatores cognitivos, fatores interpessoais, o envolvimento do paciente no tratamento, a atitude do paciente e as diferenças culturais ${ }^{13}$.

Apesar de a idade não ter apresentado diferença estatística quando feita comparação entre os gêneros masculino e feminino, pôde-se 
observar que a média de idade dos pacientes que se apresentaram no ambulatório de fisioterapia foi de 56 anos, portanto uma média elevada. Esse fato corrobora com outros estudos que relacionam a idade como fator de risco para o desenvolvimento do câncer ${ }^{14,15}$ e ainda com estudos que associam o tratamento fisioterapêutico no pós-operatório do câncer de mama em uma população com a faixa etária aproximada com a encontrada nos resultados do presente estudo ${ }^{16}$.

Em contrapartida, apesar de a idade não ter apresentado diferença entre os gêneros, outra característica da população atendida que apresentou diferença significativa foi o IMC, o que era esperado devido à diferença significativa também encontrada no gênero dos pacientes. Considera-se que taxas elevadas de IMC estejam relacionadas ao aumento do risco para desenvolvimento do câncer ${ }^{15}$, como apontado no estudo de Montazari, et $\mathrm{al}^{17}$, que verificou diferença significativa $(p=0,004)$ no IMC do grupo com câncer de mama que apresentou média de 27,9 $\pm 3,9 \mathrm{~kg} / \mathrm{m}^{2}$ comparado à média do IMC do grupo controle de $26,3 \pm 4,3 \mathrm{~kg} / \mathrm{m}^{2}$.

Os dados de gênero, IMC e idade corroboram com Ricci, et $\mathrm{al}^{10}$, que verificaram 196 muIheres brasileiras com câncer de mama, tendo a idade comparável à amostra do presente estudo, assim como IMC, que mostrou sobrepeso e obesidade, tendo a quimioterapia em determinados regimes como fator associado ao aumento de peso.

A escolaridade foi uma variável difícil de ser analisada devido à grande quantidade de dados não informados ( $\mathrm{n}=34,36,2 \%$ ). No entanto, a escolaridade da população atendida se descreve em que a maioria tinha fundamental completo ( $\mathrm{n}$ $=21,22,3 \%$ ), seguido do ensino fundamental incompleto $(n=17,18,1 \%)$. Esse valor está abaixo dos valores apresentados pelo IBGE no censo de 2010, em que 41,9 \% da população de São Paulo teria ensino fundamental incompleto, demonstrando que a amostra estudada tem baixo grau de escolaridade em relação à população da cidade de São Paulo em geral ${ }^{18}$. Machado e Nogueira ${ }^{11}$ avaliaram a satisfação dos usuários de serviços de Fisioterapia em Teresina $(\mathrm{PI})$, com relação à agilidade para agendamento da consulta e atendimento, acolhimento, confiança, ambiência, humanização, acessibilidade, eficácia e expectativa sobre os serviços recebidos. Os usuários, na sua maioria, eram mulheres $(62,5 \%)$, com uma média de idade de 49,5 anos $(<0,001)$, casados, analfabetos, dados que se assemelham aos encontrados no presente estudo.

Com relação ao diagnóstico médico e considerando que a maioria dos prontuários avaliados era de pacientes do gênero feminino, os dados do presente estudo corroboram com dados epidemiológicos que apontam o câncer de mama como a principal neoplasia maligna feminina no Brasil, e a principal causa de mortalidade entre as mulheres².

Com relação ao tempo de evolução da doença, esse se referiu ao período transcorrido entre o conhecimento do diagnóstico até o início da fisioterapia. A maioria dos pacientes (33\%) compareceu ao ambulatório de fisioterapia com tempo de evolução da doença entre 2 e 5 anos, mas não se pode afirmar com exatidão quanto tempo após o tratamento cirúrgico os pacientes iniciaram o tratamento fisioterapêutico, sendo uma limitação em nossos dados. No entanto, sabe-se que a atuação fisioterapêutica mais precoce no pós-operatório de pacientes submetidas às mastectomias pode prevenir uma série de complicações e garantir uma recuperação mais rápida ${ }^{16}$. Por meio desse achado, pode-se observar a necessidade de adequação no atendimento a pacientes em fase pós-operatória, questão observada em muitos serviços do SUS ${ }^{19}$. Como proposta, sugere-se a distribuição de cartilhas de orientação e palestras informativas quanto aos cuidados pré e pós-operatórios, bem como o de encaminhamento médico mais precoce à fisioterapia, e deve-se considerar que é comum o desenvolvimento de sequelas como o linfedema mais tardiamente, levando o paciente a procurar a fisioterapia somente após sua instalação ${ }^{20}$.

As técnicas cirúrgicas para o tratamento do câncer de mama evoluíram com o tempo e estão cada vez menos invasivas. Com isso, o tratamento cirúrgico tem sido mais indicado para a remoção do nódulo mamário, sem um tempo longo de cirurgia ${ }^{21}$, como observado em nossos resultados, que apontaram o tratamento cirúrgico como o mais realizado ( $\mathrm{n}=89,94,7 \%$ ). Apesar dos avanços no tratamento cirúrgico do câncer de mama, 
observam-se ainda diversas complicações no pós-operatório: episódios de infecção do braço, seroma, necrose tecidual, sintomas sensoriais do nervo intercostobraquial, escápula alada e redução da amplitude de movimento do ombro ${ }^{20}$.

A alta taxa de procedimento cirúrgico realizado na amostra deste estudo pode estar associada ao diagnóstico fisioterapêutico mais frequente, que foi de alterações vasculares e linfáticas, tendo destaque o linfedema de membros superiores ( $n=42,51 \%, p<0,001)$. Estudo de Bergmann, et $\mathrm{al}^{20}$ avaliou a prevalência de linfedema em mulheres com diagnóstico de câncer de mama e verificou que existem alguns fatores que podem estar associados com o desenvolvimento do linfedema, como a radioterapia na região axilar, obesidade, redução da amplitude de movimento do ombro e episódios prévios de infecção do membro superior.

A queixa mais relatada foi a dor $(\mathrm{n}=52$, $55,3 \%)$, seguida do edema ( $n=48,51,1 \%)$. A dor no paciente com câncer pode estar relacionada aos tratamentos associados, como, por exemplo, a quimioterapia ${ }^{22}$, ou, ainda, às complicações causadas pelo câncer, como, por exemplo, a compressão dos nociceptores, presença de linfedema de membro superior, redução da mobilidade articular do ombro ${ }^{23}$, entre outros fatores, como as complicações emocionais e psicossomáticos ${ }^{24}$.

Por esse motivo, a atuação do fisioterapeuta deve objetivar a reabilitação física e a promoção de qualidade de vida, reduzindo sintomas e queixas relatados pelos pacientes com diagnóstico de câncer ${ }^{16}$.

A avaliação fisioterapêutica realizada por diferentes profissionais ao longo dos dois anos da coleta de dados pode ser considerada uma limitação no estudo. Devemos considerar que protocolos de atendimento e conduta são específicos de cada serviço ou setor de atendimento fisioterapêutico. Dessa maneira, os resultados obtidos podem ser diferentes de outros centros de atendimento aos pacientes com câncer. Vale ressaltar a importância de se conhecerem os diferentes perfis dos pacientes para que o atendimento da fisioterapia seja aprimorado e especializado.

Sugere-se que novos estudos sejam realizados na tentativa de se verificar a demanda e perfil de pacientes atendidos em ambulatórios de fisioterapia em oncologia, como estudos coorte prospectivos, a fim de se evitar os vieses da metodologia retrospectiva. Apesar dessa limitação, os dados do presente estudo podem ser utilizados como base para estudos futuros, assim como para correção e/ou implementação de serviços de fisioterapia que tenham abrangência mais completa ao paciente com câncer.

\section{CONCLUSÃO}

O perfil clínico dos pacientes atendidos no ambulatório de fisioterapia caracterizou-se por pacientes do gênero feminino, faixa etária acima de 50 anos e com sobrepeso. A maioria tinha ensino fundamental completo, diagnóstico de câncer de mama e vinha para o tratamento fisioterapêutico entre o segundo e o quinto ano de evolução da doença e depois de realizarem o tratamento cirúrgico. As alterações vasculares e linfáticas são as mais frequentes associadas às queixas de dor e edema. Essa caracterização dos pacientes que necessitam de atendimento fisioterapêutico em um instituto de referência faz-se necessário para a estruturação do serviço, incluindo a capacitação de profissionais especializados para o atendimento. Tais informações podem direcionar possibilidades terapêuticas, permitindo maior efetividade no tratamento.

\section{REFERÊNCIAS}

1. OMS. Organização Mundial de Saúde. World Cancer Report, released Tuesday by the World Health Organization; 2008.

2. INCA. Instituto Nacional de Câncer José Alencar Gomes da Silva. Estimativa 2014: incidência de câncer no Brasil [internet]. Rio de Janeiro: INCA; 2014. 118 p.

3. Souza RJSP, Mattedi AP, Corrêa MP, Rezende ML, Ferreira ACA. Estimativa do custo do tratamento do câncer de pele tipo não-melanoma no Estado de São Paulo - Brasil. An Bras Dermato. 2011;86(4):657-62. DOI: $\underline{\text { http://dx.doi.org/10.1590/ }}$ s0365-05962011000400005. 
4. Stout NL. Cancer prevention in physical therapist practice. Phys Ther. 2009;89(11):1119-22. DOI: http://dx.doi. org/10.2522/ptj.2009.89.11.1119.

5. Hashibe M, Hunt J, Wei M, Buys S, Gren L, Lee YC. Tobacco, alcohol, body mass index, physical activity, and the risk of head and neck cancer in the prostate, lung, colorectal, and ovarian (PLCO) cohort. Head Neck. 2013;35(7):914-22. DOI: http://dx.doi.org/10.1002/hed.23052.

6. Dean E. Physical therapy in the 21st century (Part I): toward practice informed by epidemiology and the crisis of lifestyle conditions. Physiother Theory Pract. 2009;25(5-6):330-53. DOI: http://dx.doi.org/10.1080/09593980802668027.

7. Tacani PM, Franceschini JP, Tacani RE, Machado AF, Montezello D, Góes JC, et al. Retrospective study of the physical therapy modalities applied in head and neck lymphedema treatment. Head Neck. DOI: http://dx.doi.org/10.1002/ hed.23899.

8. Tonezzer T, Tagliaferro J, Cocco M, Marx A. Uso da estimulação elétrica nervosa transcutânea aplicado ao ponto de acupuntura PC6 para a redução dos sintomas de náusea e vômitos associados à quimioterapia antineoplásica. Rev Bras Cancerol. 2012;58(1):7-14.

9. COFFITO. Conselho Federal de Fisioterapia e Terapia Ocupacional. Especialidades reconhecidas pelo Coffito.

10. Ricci MD, Formigoni MC, Zuliani LM, Aoki DS, Mota BS, Filassi JR, et al. Variations in the body mass index in Brazilian women undergoing adjuvant chemotherapy for breast cancer. Rev Bras Ginecol Obstet. 2014;36(11):503-8. DOI: http:// dx.doi.org/10.1590/s0100-720320140005081.

11. Machado NP, Nogueira LT. Avaliação da satisfação dos usuários de serviços de Fisioterapia. Rev Bras Fisioter. 2008;12(5):401-8. DOI: http://dx.doi.org/10.1590/s1413-35552008000500010.

12. Dent SF, Visram H, Kanji F. Endocrine therapy for male breast cancer: rates of toxicity and adherence. Curr Oncol. 2010;17(5):17-21. DOI: http://dx.doi.org/10.3747/co.v17i5.631.

13. Huiart L, Bardou VJ, Giorgi R. The importance of adherence to oral therapies in the field of oncology: the example of breast cancer. Bull Cancer. 2013;100(10):1007-15.

14. Matos JC, Pelloso SM, Carvalho MDB. Prevalência de fatores de risco para o câncer de mama no município de Maringá, Paraná. Rev Latino-Am Enf. 2010;18(3):57-64.

15. Namiranian N, Moradi-Lakeh M, Razavi-Ratki SK, Doayie M, Nojomi M. Risk factors of breast cancer in the eastern mediterranean region: a systematic review and meta-analysis. Asian Pac J Cancer Prev. 2014;15(21):9535-41.

16. Amaral MTP, Oliveira MMF, Ferreira NO, Guimarães RV, Sarian LO, Gurgel MSC. Manual therapy associated with upper limb exercises vs. exercises alone for shoulder rehabilitation in postoperative breast cancer. Physiother Theory Pract. 2012;28(4):299-306. DOI: http://dx.doi.org/10.3109/09593985.2011.604709.

17. Montazeri A, Sadighi J, Farzadi F, Maftoon F, Vahdaninia M, Ansari M, et al. Weight, height, body mass index and risk of breast cancer in postmenopausal women: a case-control study. BMC Cancer. 2008;8(1):278. DOI: http://dx.doi. org/10.1186/1471-2407-8-278.

18. IBGE. Instituto Brasileiro de Geografia e Estatística; 2014.

19. Siqueira FV, Facchini LA, Hallal PC. Epidemiology of physiotherapy utilization among adults and elderly. Rev Saúde Pública. 2005;39(4):663-8. DOI: http://dx.doi.org/10.1590/s0034-89102005000400022.

20. Bevilacqua JLB, Kattan MW, Changhong Y, Koifman S, Mattos IE, Koifman RJ, Bergmann A. Nomograms for predicting the risk of arm lymphedema after axillary dissection in breast cancer. Ann Surg Oncol. 2012;19(8):2580-9. DOI: http:// dx.doi.org/10.1245/s10434-012-2290-x.

21. Zervoudis S, latrakis G, Mares P, Boileau L, Grammatikakis I, Evangelinakis N, et al. Breast conserving surgery in multicentric breast cancer, preliminary data of our experience. Eur J Gynaecol Oncol. 2014;35(5):530-4.

22. Costa AIS, Chaves MD. Dor em pacientes oncológicos sob tratamento quimioterápico. Rev Dor. 2012;13(1):45-9. DOI: http://dx.doi.org/10.1590/s1806-00132012000100008.

23. Khan F, Amatya B, Pallant JF, Rajapaksa I. Factors associated with long-term functional outcomes and psychological sequelae in women after breast cancer. Breast J. 2012;21(3):314-20. DOI: http://dx.doi.org/10.1016/j.breast.2012.01.013. 24. Langford DJ, Paul SM, West C, Abrams G, Elboim C, Levine JD, et al. Persistent arm pain is distinct from persistent breast pain following breast cancer surgery. J Pain. 2014;15(12):1238-47. 